\title{
Estimating cases of severe malaria at the population-level: Analysis of household surveys from 19 malaria endemic countries in Africa
}

\author{
Cameron Taylor ( $\square$ cameron.taylor@icf.com ) \\ ICF https://orcid.org/0000-0002-5474-0835 \\ Sorrel ML NAMASTE \\ ICF International Inc \\ Joanna LOWELL \\ Vysnova Partners \\ Johanna USEEM \\ ICF International Inc \\ Yazoumé YÉ \\ ICF International Inc
}

Research

Keywords: severe malaria, population-based surveys, Demographic and Health Surveys, Malaria Indicator Surveys, sub-Saharan Africa

Posted Date: June 4th, 2020

DOI: https://doi.org/10.21203/rs.3.rs-32066/v1

License: (c) (i) This work is licensed under a Creative Commons Attribution 4.0 International License. Read Full License 


\section{Abstract \\ Background}

The burden of severe malaria is uncertain at the population level because existing estimates rely exclusively on data from the formal healthcare system. Using data from population-based surveys this analysis examines severe malaria cases at the population level, which captures children whose caregivers 1) have taken the child to a healthcare facility but the child's illness did not resolve, or 2) have not sought care for the child's illness. Direct inclusion of these children in severe malaria estimates has been an underlying data gap.

\section{Methods}

This analysis examined data from 37 Demographic and Health Surveys and Malaria Indicator Surveys across 19 countries in sub-Saharan Africa collected between 2011 and 2018. The outcome of interest is severe malaria, defined as children age 6-59 months who were positive for malaria with at least one self-reported symptom for severe malaria, including loss of consciousness, rapid breathing, seizures, or severe anemia. The study includes a weighted descriptive, country-level analysis and a multilevel mixed-effects logistic regression model to assess the determinants of severe malaria.

\section{Results}

Among children positive for malaria across all surveys, 4.5\% (95\% Cl 4.1-4.8) had at least one symptom of severe malaria, which was significantly associated with age, residence, wealth, and survey timing at a p-value less than 0.05 . Children in the higher malaria transmission zone were more likely to have symptoms compared to those in the lowest transmission zone; however, these results were not statistically significant.

\section{Conclusion}

An accurate estimate of the burden of severe malaria is essential to assessing the impact of malaria interventions and to guiding future malaria investments. This analysis presents a novel approach of estimating the burden of severe malaria in children under age five in malaria endemic countries. Estimating severe malaria through household-based surveys allows countries to estimate severe malaria across time and to compare with other countries. Having a population level estimate of severe malaria helps further our understanding of the burden and epidemiology of severe malaria.

\section{Background}

In 2018, an estimated 405,000 deaths from malaria occurred globally, with an estimated $70 \%$ of all malaria deaths occurring in children under age 5 in sub-Saharan Africa (SSA) [1]. Malaria typically begins as an acute febrile illness. If not appropriately treated, Plasmodium falciparum (P. falciparum) malaria can progress to severe illness, and can often lead to death. Children with severe malaria frequently develop one or more of the following complications: severe anemia, respiratory distress, or cerebral malaria [2, 3]. The clinical manifestations of these severe malaria complications in children include impaired consciousness, respiratory distress (acidotic breathing), multiple convulsions, prostrations, shock, and jaundice [2, 4]. The clinical epidemiology of severe malaria can also present differently according to age and transmission intensity. Studies have shown that as the intensity of malaria transmission increases, the mean age of severe malaria decreases[5-8]. In high transmission areas, the risk for severe malaria is greatest among young children (the first few months of life to age 5). Severe malaria becomes less common in older children when acquired immunity provides a protective effect. Conversely, in low transmission areas, severe malaria is more common in older children and adults [7].

Although the clinical features and transmission patterns of severe malaria are well understood, accurately capturing the burden of severe malaria remains a challenge. First, severe malaria symptoms are non-specific, which makes it difficult to differentiate severe malaria cases from other diseases that are also common in malaria-endemic countries [2]. Second, severe malaria case estimates from routine data collected through formal healthcare services are often affected by variable data quality and non-standard case definitions, which make comparisons across countries challenging [9-11]. Finally, a proportion of severe malaria illnesses and deaths occur outside the formal healthcare system and are therefore undocumented [7, 9, 12-14]. 
An accurate estimate of the burden of severe malaria is essential to assess the impact of malaria prevention and control interventions and to guide future malaria investments. To date, the only robust estimates of severe malaria cases include children who present to the formal healthcare system or data from small epidemiological studies across SSA. It has been a challenge to use these data to model the number of severe malaria cases across SSA because of varying age ranges of reporting, different diagnosis techniques, surveillance methods, and health care utilization $[5,8]$. The Demographic and Health Surveys (DHS) Program, the primary global source of population-based malaria data, tests children for malaria and also measures hemoglobin concentration to test for anemia. For children who test positive for malaria, The DHS Program collects data on severe malaria symptoms for referral purposes during data collection. However, this information has not been used to examine signs and symptoms of severe malaria. To better understand the burden of severe malaria in SSA, this paper presents an analysis of severe malaria symptoms in children age 6-59 months who are positive for $P$. falciparum malaria, and whose data are captured in population-based surveys.

\section{Methods}

\section{Data on severe malaria}

This study used data from DHS and Malaria Indicator Surveys (MIS), which are both nationally representative, population-based household surveys of The DHS Program [15]. All surveys are independent but use standardized data collection procedures and tools. When requested by the country, the DHS and MIS collect blood samples for anemia and malaria testing among children age 6-59 months. All children are tested regardless of whether they have signs or symptoms of malaria. Specially trained biomarker health technicians (who are usually nurses) take capillary blood obtained with a finger or heel prick. The blood is immediately tested for anemia and malaria in the field and the results are provided to respondents' parents or guardians within a few minutes.

In the field, biomarker health technicians use rapid diagnostic tests (RDT) to determine if children have malaria. The RDTs detect the histidine-rich protein II (HRP-II) antigen of $P$. falciparum in blood. P. falciparum is the primary cause of severe malaria and is the predominant species found in the countries included in this analysis[2, 7]. Often blood is also collected for the examination of blood smears by microscopy in the laboratory. DHS and MIS surveys also typically test children age 6-59 months for anemia with capillary blood collected through a finger or heel prick. The tests use the HemoCue $201+$ and, on occasion, the HemoCue 301 point-of-care hemoglobin testing system.

When the DHS and MIS surveys first introduced malaria testing in 2006, biomarker health technicians offered antimalarial treatment to all children with positive RDT results. Beginning in 2011, The DHS Program instituted a referral system in which children who are positive for malaria but have no signs and symptoms of severe illness receive antimalarial treatment, and children with signs and symptoms of severe illness are referred to the nearest health facility for treatment.

At that time, additional questions were added to the survey's questionnaire to screen children for signs and symptoms of severe malaria. To assess severe malaria signs and symptoms, the caregiver is asked: "Does (NAME) suffer from any of the following illnesses or symptoms: extreme weakness, heart problems, inability to drink or breastfeed, vomiting everything, loss of consciousness, rapid breathing, seizures, bleeding, jaundice, or dark urine?" If the child is positive for malaria (by RDT) and the caregiver answers "Yes" to any of the signs and symptoms and/or if the child has a hemoglobin concentration of $<8$ grams per deciliter $(\mathrm{g} / \mathrm{dL})$, the child is given a referral slip for severe malaria to take to the nearest healthcare facility for care[16]. A child who is negative for malaria (by RDT) is not asked about severe malaria symptoms. (See additional file 1 for more information about The DHS Program malaria referral process.)

\section{Data analysis}

\section{Case definition of severe malaria}

This analysis used a severe malaria case definition based on signs and symptoms captured in DHS and MIS surveys that most closely aligns with the clinical symptoms of malaria outlined in the World Health Organization (WHO) Management of Severe Malaria Handbook [2]. Of the 11 severe malaria signs and symptoms collected by DHS and MIS surveys, loss of consciousness, rapid breathing, seizures, or severe anemia (hemoglobin levels $<5 \mathrm{~g} / \mathrm{dL}$ adjusted for altitude) were the four most common clinical manifestations of severe malaria in children that most closely align with the symptoms identified in the WHO Management of Severe Malaria Handbook. Hemoglobin levels are adjusted for the altitude in areas that are above 1,000 meters in elevation [17].

\section{Study variables}


Outcome variable: severe malaria. The outcome of interest is severe malaria, defined as children age 6-59 months who were positive for malaria with at least one symptomatic marker for severe malaria, including loss of consciousness, rapid breathing, seizures, or severe anemia. The authors calculated the percentage of children age 6-59 months with severe malaria of all children age 6-59 months who were positive for malaria, according to RDT.

Covariates: all potential confounders. For this analysis, variables found in the literature related to severe malaria were reviewed and included based on data availability. The model included the following covariates: sex, age, place of residence, wealth quintiles, malaria endemicity, survey timing, and country (Table 1). The child's age was divided into four age categories: 6-23 months, 24-35 months, 3647 months, and 48-59 months. Place of residence is defined as whether a household is located in a rural or urban area. Wealth quintiles were derived from the DHS wealth index, which measures the relative socioeconomic status of households based on household assets and amenities at a point in time [18]. Survey timing is divided into two equal categories based on the fieldwork dates of 2011-2014 and 20152018. To determine malaria endemicity, we assigned each child's household enumeration area into geographical zones based on malaria transmission risk. To link the DHS and MIS geo-coordinates (latitude, longitude) of each survey enumeration area to transmission risk zones, we used geo-coordinated $P$. falciparum parasite prevalence rates among children age $2-10\left(P f P R_{2}-10\right)$ from the Malaria Atlas Project 2015 [19]. We assigned every child's household in an enumeration area from the DHS or MIS survey dataset to the same malaria transmission risk zone based on corresponding $P \mathrm{PPR}_{2-10}$ data for that enumeration area. For the transmission zone categories, we used risk categories as outlined in the WHO Management of Severe Malaria Handbook [2] with low transmission defined as PfPR $2-10 \leq 10 \%$ and high transmission defined as $\mathrm{PfPR}_{2-10}>50 \%$. Due to a high number of children in the moderate risk category $(11 \%<P f \mathrm{PR} 2-10 \leq 50 \%)$, we divided this risk group into two equal risk categories of moderate transmission $\mathrm{A}\left(11 \%<P f P R_{2}-10 \leq 30 \%\right)$ and moderate transmission $\mathrm{B}$ $\left(31 \%<P f R_{2-10} \leq 50 \%\right)$.

Table 1

Descriptions of co-variates included in the analysis

\begin{tabular}{|c|c|c|c|}
\hline Variable & Type & Option & Details of Measurement \\
\hline Sex & Categorical & Two categories: male, female & Collected from the household questionnaire \\
\hline \multirow[t]{2}{*}{ Age Group } & \multirow[t]{2}{*}{ Categorical } & Four categories (months): & \multirow{2}{*}{$\begin{array}{l}\text { Based on date of birth and date of interview from } \\
\text { the household questionnaire }\end{array}$} \\
\hline & & $6-23,24-35,36-47,48-59$ & \\
\hline $\begin{array}{l}\text { Place of } \\
\text { Residence }\end{array}$ & Categorical & Two categories: urban and rural & $\begin{array}{l}\text { Household classified as being in an urban or rural } \\
\text { area }\end{array}$ \\
\hline $\begin{array}{l}\text { Wealth } \\
\text { Quintile }\end{array}$ & Categorical & $\begin{array}{l}\text { Five categories: Lowest, Second, Middle, Fourth, } \\
\text { Highest }\end{array}$ & Asset-based principal component analysis \\
\hline $\begin{array}{l}\text { Malaria } \\
\text { Endemicity }\end{array}$ & Categorical & $\begin{array}{l}\text { Four categories: low risk, intermediate risk } A \text {, } \\
\text { intermediate risk } B \text {, and high risk }\end{array}$ & $\begin{array}{l}\text { Categorized using Malaria Atlas Project (MAP) } \\
\text { PfPR }_{2-10} \text { values }\end{array}$ \\
\hline $\begin{array}{l}\text { Survey } \\
\text { Timing }\end{array}$ & Categorical & Two categories: 2011-2014 and 2015-2018 & Based on year of survey fieldwork \\
\hline Country & Categorical & $\begin{array}{l}19 \text { categories representing each of the country } \\
\text { included in the study }\end{array}$ & Based on survey country \\
\hline
\end{tabular}

\section{Study population}

The inclusion criteria for the study were all malaria-endemic countries in SSA that have conducted a DHS or MIS survey in which children were tested for malaria and anemia, and the surveys included questions on signs or symptoms of severe malaria. In total, this analysis examined results from 37 surveys across 19 countries (Fig. 1). The study population for this analysis included children age $6-59$ months who stayed in surveyed households the night before the survey and received $P$. falciparum malaria parasite (RDT) and anemia tests. Children with any missing household enumeration area $\mathrm{PfPR}_{2-10}$ data were excluded from the analysis.

\section{Regression analysis}

The study includes a country-level descriptive analysis weighted for complex survey design and a multi-country weighted pooled analysis, both with 95\% confidence intervals. The study used a multilevel (individual-level and country-level) unweighted mixed-effects logistic regression model to assess the determinants of severe malaria. The model includes sex, age of the child, residence, household wealth, malaria transmission zones, and survey timing. All analyses were conducted with StataSE16 (StataCorp LP, College Station, USA). 


\section{Results}

\section{Descriptive Analysis}

\section{Country-level analysis}

A total of 183,265 children who met the inclusion criteria were included in the study of the 209,216 children age $6-59$ months eligible for malaria and anemia testing. The overall prevalence of malaria infection in children age 6-59 months, as detected with RDTs, across all surveys was $26.0 \%$, ranging from $0.6 \%$ (Senegal DHS 2015) to 61.4\% (Burkina Faso MIS 2014). Among children positive for malaria across all surveys, $4.5 \%$ (95\% $\mathrm{Cl} 4.1-4.8)$ of children had at least one severe malaria symptom (loss of consciousness, seizures, rapid breathing, or severe anemia), ranging from <0.1\% in the Senegal DHS 2015 to 17.4\% in the Madagascar MIS 2011. The prevalence of individual symptoms varies by country. Mali DHS 2018 (2.3\%) had the highest percentage of children with loss of consciousness, Guinea DHS 2012 (9.3\%) the highest percentage of children with seizures, Madagascar MIS $2011(14.2 \%)$ the highest percentage of children with rapid breathing, and Senegal DHS 2016 (3.2\%) the highest percentage of children with severe anemia (Table 2). 
Table 2

Percentage of children age 6-59 months with a malaria infection according to RDT, and among those with a malaria infection, the percentage with specified severe malaria symptoms, and the percentage with at least one severe malaria symptom, according to country and survey

\begin{tabular}{|c|c|c|c|c|c|c|c|c|c|c|}
\hline & & & & Among children & with a mala & la infection & & & & \\
\hline & & & & Percentage with & specified se & ere malaria & symptoms & & & \\
\hline & $\begin{array}{l}\text { Perce } \\
\text { childr } \\
\text { a mal } \\
\text { infect }\end{array}$ & $\begin{array}{l}\text { tage of } \\
\text { n with } \\
\text { ria } \\
\text { on }\end{array}$ & $\begin{array}{l}\text { Number } \\
\text { of } \\
\text { children } \\
\text { tested } \\
\text { for }\end{array}$ & $\begin{array}{l}\text { Loss of } \\
\text { consciousness }\end{array}$ & Seizures & $\begin{array}{l}\text { Rapid } \\
\text { breathing }\end{array}$ & $\begin{array}{l}\text { Severe } \\
\text { anemia } \\
\text { (Hemoglobin } \\
<5 \text { grams } \\
\text { per deciliter) }\end{array}$ & $\begin{array}{l}\text { Perce } \\
\text { with a } \\
\text { one s } \\
\text { mala } \\
\text { symp }\end{array}$ & $\begin{array}{l}\text { tage } \\
\text { least } \\
\text { vere } \\
\text { om }\end{array}$ & $\begin{array}{l}\text { Number } \\
\text { of } \\
\text { children } \\
\text { with } \\
\text { malaria }\end{array}$ \\
\hline Country/Survey & $\%$ & $\begin{array}{l}95 \% \\
\mathrm{Cl}\end{array}$ & $\mathbf{N}$ & $\%$ & $\%$ & $\%$ & $\%$ & $\%$ & $\begin{array}{l}95 \% \\
\mathrm{Cl}\end{array}$ & $\mathbf{N}$ \\
\hline $\begin{array}{l}\text { Angola DHS } \\
2015-16\end{array}$ & $13 \cdot 6$ & $\begin{array}{l}(11 \cdot 8- \\
15 \cdot 6)\end{array}$ & 6,552 & 1 & $0 \cdot 8$ & $8 \cdot 8$ & $2 \cdot 6$ & $11 \cdot 6$ & $\begin{array}{l}(7 \cdot 5- \\
17 \cdot 3)\end{array}$ & 891 \\
\hline $\begin{array}{l}\text { Benin DHS } \\
\text { 2017-18 }\end{array}$ & $36 \cdot 5$ & $\begin{array}{l}(34 \cdot 1- \\
38 \cdot 9)\end{array}$ & 6,035 & $0 \cdot 2$ & 0.5 & $1 \cdot 1$ & $0 \cdot 4$ & $2 \cdot 1$ & $\begin{array}{l}(1 \cdot 5- \\
3 \cdot 0)\end{array}$ & 2,200 \\
\hline $\begin{array}{l}\text { Burkina Faso } \\
\text { MIS } 2014\end{array}$ & $61 \cdot 4$ & $\begin{array}{l}(58 \cdot 5- \\
64 \cdot 2)\end{array}$ & 5,743 & 0.5 & 0.7 & $1 \cdot 7$ & $1 \cdot 1$ & $3 \cdot 6$ & $\begin{array}{l}(2 \cdot 9- \\
4 \cdot 5)\end{array}$ & 3,524 \\
\hline $\begin{array}{l}\text { Burkina Faso } \\
\text { MIS } 2017\end{array}$ & $20 \cdot 1$ & $\begin{array}{l}(17 \cdot 8- \\
22 \cdot 5)\end{array}$ & 5,042 & 0.5 & $1 \cdot 7$ & 1.9 & $0 \cdot 8$ & $3 \cdot 6$ & $\begin{array}{l}(2 \cdot 4- \\
5 \cdot 3)\end{array}$ & 1,012 \\
\hline $\begin{array}{l}\text { Burundi MIS } \\
2012\end{array}$ & $22 \cdot 2$ & $\begin{array}{l}(18 \cdot 1- \\
27 \cdot 0)\end{array}$ & 3,774 & $2 \cdot 0$ & $2 \cdot 8$ & $9 \cdot 0$ & $1 \cdot 6$ & $10 \cdot 2$ & $\begin{array}{l}(7 \cdot 7- \\
13 \cdot 4)\end{array}$ & 838 \\
\hline $\begin{array}{l}\text { Burundi DHS } \\
2016-2017\end{array}$ & $38 \cdot 7$ & $\begin{array}{l}(36 \cdot 2- \\
41 \cdot 3)\end{array}$ & 5,639 & 0.6 & 1 & $3 \cdot 3$ & $1 \cdot 1$ & $4 \cdot 3$ & $\begin{array}{l}(3 \cdot 4- \\
5 \cdot 4)\end{array}$ & 2,184 \\
\hline $\begin{array}{l}\text { DRC DHS } \\
2013-14\end{array}$ & $31 \cdot 0$ & $\begin{array}{l}(28 \cdot 0- \\
34 \cdot 3)\end{array}$ & 7,329 & 0.9 & $2 \cdot 2$ & $5 \cdot 0$ & 0.8 & $7 \cdot 3$ & $\begin{array}{l}(5 \cdot 7- \\
9 \cdot 3)\end{array}$ & 2,276 \\
\hline $\begin{array}{l}\text { Ghana DHS } \\
2014\end{array}$ & $36 \cdot 3$ & $\begin{array}{l}(32 \cdot 7- \\
40 \cdot 0)\end{array}$ & 2,495 & $<0.1$ & $0 \cdot 3$ & 0.7 & 0.3 & $1 \cdot 0$ & $\begin{array}{l}(0 \cdot 6- \\
1 \cdot 9)\end{array}$ & 905 \\
\hline $\begin{array}{l}\text { Ghana MIS } \\
2016\end{array}$ & $28 \cdot 1$ & $\begin{array}{l}(23 \cdot 7- \\
33 \cdot 0)\end{array}$ & 2,624 & $<0.1$ & $0 \cdot 4$ & 0.5 & $1 \cdot 0$ & $1 \cdot 7$ & $\begin{array}{l}(0 \cdot 9- \\
3 \cdot 3)\end{array}$ & 739 \\
\hline $\begin{array}{l}\text { Guinea DHS } \\
2012\end{array}$ & $48 \cdot 0$ & $\begin{array}{l}(44 \cdot 1- \\
51 \cdot 9)\end{array}$ & 3,144 & 0.5 & $9 \cdot 3$ & $5 \cdot 7$ & $1 \cdot 1$ & $15 \cdot 6$ & $\begin{array}{l}(11 \cdot 7- \\
20 \cdot 6)\end{array}$ & 1,510 \\
\hline $\begin{array}{l}\text { Kenya MIS } \\
2015\end{array}$ & $9 \cdot 9$ & $\begin{array}{l}(7.7- \\
12.7)\end{array}$ & 2,790 & $<0.1$ & $<0.1$ & 0.3 & $<0.1$ & 0.3 & $\begin{array}{l}(0 \cdot 1- \\
1 \cdot 8)\end{array}$ & 277 \\
\hline $\begin{array}{l}\text { Liberia MIS } \\
2011\end{array}$ & $45 \cdot 3$ & $\begin{array}{l}(41 \cdot 6- \\
49 \cdot 1)\end{array}$ & 2,835 & 0.3 & 0.7 & 0.7 & $0 \cdot 2$ & $1 \cdot 7$ & $\begin{array}{l}(1 \cdot 0- \\
3 \cdot 0)\end{array}$ & 1,285 \\
\hline $\begin{array}{l}\text { Liberia MIS } \\
2016\end{array}$ & $45 \cdot 3$ & $\begin{array}{l}(41 \cdot 3- \\
49 \cdot 4)\end{array}$ & 2,822 & $0 \cdot 1$ & 1 & 1.9 & 0.5 & $2 \cdot 9$ & $\begin{array}{l}(1.9- \\
4 \cdot 4)\end{array}$ & 1,278 \\
\hline $\begin{array}{l}\text { Madagascar } \\
\text { MIS } 2011\end{array}$ & $8 \cdot 8$ & $\begin{array}{l}(6 \cdot 9- \\
11 \cdot 1)\end{array}$ & 6,188 & $1 \cdot 0$ & $3 \cdot 7$ & $14 \cdot 2$ & $<0.1$ & $17 \cdot 4$ & $\begin{array}{l}(11 \cdot 7- \\
25 \cdot 2)\end{array}$ & 542 \\
\hline $\begin{array}{l}\text { Madagascar } \\
\text { MIS } 2013\end{array}$ & $10 \cdot 1$ & $\begin{array}{l}(8 \cdot 0- \\
12 \cdot 8)\end{array}$ & 5,490 & $1 \cdot 8$ & $4 \cdot 8$ & $5 \cdot 3$ & 0.4 & $9 \cdot 2$ & $\begin{array}{l}(6 \cdot 0- \\
13 \cdot 7)\end{array}$ & 557 \\
\hline $\begin{array}{l}\text { Madagascar } \\
\text { MIS } 2016\end{array}$ & $5 \cdot 2$ & $\begin{array}{l}(3 \cdot 6- \\
7 \cdot 5)\end{array}$ & 6,480 & $<0.1$ & $0 \cdot 1$ & $2 \cdot 1$ & 0.4 & $2 \cdot 5$ & $\begin{array}{l}(0 \cdot 9- \\
6 \cdot 8)\end{array}$ & 337 \\
\hline $\begin{array}{l}\text { Malawi MIS } \\
2012\end{array}$ & $43 \cdot 4$ & $\begin{array}{l}(37 \cdot 7- \\
49 \cdot 2)\end{array}$ & 2,182 & $1 \cdot 4$ & 0.7 & $5 \cdot 1$ & $1 \cdot 1$ & 6.9 & $\begin{array}{l}(4 \cdot 6- \\
10 \cdot 3)\end{array}$ & 946 \\
\hline $\begin{array}{l}\text { Malawi MIS } \\
2014\end{array}$ & $37 \cdot 1$ & $\begin{array}{l}(30 \cdot 1- \\
44 \cdot 6)\end{array}$ & 1,991 & $0 \cdot 1$ & $<0.1$ & $1 \cdot 2$ & 0.2 & $1 \cdot 4$ & $\begin{array}{l}(0 \cdot 6- \\
3 \cdot 1)\end{array}$ & 738 \\
\hline $\begin{array}{l}\text { Malawi MIS } \\
2017\end{array}$ & $36 \cdot 1$ & $\begin{array}{l}(31 \cdot 3- \\
41 \cdot 2)\end{array}$ & 2,468 & $0 \cdot 2$ & $0 \cdot 2$ & $2 \cdot 2$ & 0.4 & $2 \cdot 8$ & $\begin{array}{l}(1 \cdot 6- \\
4 \cdot 7)\end{array}$ & 892 \\
\hline
\end{tabular}




\begin{tabular}{|c|c|c|c|c|c|c|c|c|c|c|}
\hline \multirow[b]{3}{*}{ Mali MIS 2015} & \multirow[b]{3}{*}{$32 \cdot 4$} & \multirow[b]{3}{*}{$\begin{array}{l}(27 \cdot 9- \\
37 \cdot 2)\end{array}$} & \multirow[b]{3}{*}{7,080} & \multicolumn{6}{|c|}{ Among children with a malaria infection } & \multirow[b]{3}{*}{2293} \\
\hline & & & & \multicolumn{6}{|c|}{ Percentage with specified severe malaria symptoms } & \\
\hline & & & & $0 \cdot 3$ & $1 \cdot 3$ & $3 \cdot 8$ & $2 \cdot 5$ & $6 \cdot 6$ & $\begin{array}{l}(5 \cdot 1- \\
8 \cdot 5)\end{array}$ & \\
\hline Mali DHS 2018 & $19 \cdot 1$ & $\begin{array}{l}(16 \cdot 6- \\
21 \cdot 9)\end{array}$ & 4,366 & $2 \cdot 3$ & $4 \cdot 8$ & $7 \cdot 6$ & $1 \cdot 9$ & $12 \cdot 8$ & $\begin{array}{l}(9 \cdot 4- \\
17 \cdot 1)\end{array}$ & 835 \\
\hline $\begin{array}{l}\text { Mozambique } \\
\text { AIS/MIS } 2015\end{array}$ & $40 \cdot 4$ & $\begin{array}{l}(36 \cdot 5- \\
44 \cdot 4)\end{array}$ & 4,515 & $<0 \cdot 1$ & $<0.1$ & $1 \cdot 9$ & $1 \cdot 8$ & $3 \cdot 3$ & $\begin{array}{l}(2 \cdot 1- \\
5 \cdot 2)\end{array}$ & 1,824 \\
\hline $\begin{array}{l}\text { Mozambique } \\
\text { MIS } 2018\end{array}$ & $39 \cdot 1$ & $\begin{array}{l}(34 \cdot 5- \\
44 \cdot 0)\end{array}$ & 4,389 & $1 \cdot 0$ & $0 \cdot 8$ & $6 \cdot 0$ & $1 \cdot 4$ & $7 \cdot 6$ & $\begin{array}{l}(5 \cdot 4- \\
10 \cdot 7)\end{array}$ & 1,718 \\
\hline $\begin{array}{l}\text { Nigeria MIS } \\
2015\end{array}$ & $44 \cdot 6$ & $\begin{array}{l}(40 \cdot 9- \\
48 \cdot 4)\end{array}$ & 5,859 & $<0 \cdot 1$ & $0 \cdot 2$ & 0.9 & $0 \cdot 5$ & $1 \cdot 7$ & $\begin{array}{l}(1 \cdot 2- \\
2 \cdot 4)\end{array}$ & 2,615 \\
\hline $\begin{array}{l}\text { Nigeria DHS } \\
2018-19\end{array}$ & $36 \cdot 6$ & $\begin{array}{l}(34 \cdot 7- \\
38 \cdot 4)\end{array}$ & 11,043 & $0 \cdot 1$ & $0 \cdot 2$ & $0 \cdot 6$ & $0 \cdot 6$ & $1 \cdot 2$ & $\begin{array}{l}(0 \cdot 8- \\
1 \cdot 6)\end{array}$ & 4,038 \\
\hline $\begin{array}{l}\text { Senegal DHS } \\
2012-13\end{array}$ & $3 \cdot 4$ & $\begin{array}{l}(2 \cdot 5- \\
4 \cdot 6)\end{array}$ & 5,155 & $<0.1$ & $<0.1$ & 0.5 & $<0.1$ & $2 \cdot 6$ & $\begin{array}{l}(1 \cdot 3- \\
5 \cdot 2)\end{array}$ & 174 \\
\hline $\begin{array}{l}\text { Senegal DHS } \\
2014\end{array}$ & $1 \cdot 1$ & $\begin{array}{l}(0 \cdot 7- \\
1 \cdot 9)\end{array}$ & 5,351 & $<0.1$ & $<0.1$ & $<0.1$ & $0 \cdot 8$ & $0 \cdot 8$ & $\begin{array}{l}(0 \cdot 1- \\
4 \cdot 8)\end{array}$ & 61 \\
\hline $\begin{array}{l}\text { Senegal DHS } \\
2015\end{array}$ & $0 \cdot 6$ & $\begin{array}{l}(0.4- \\
0.9)\end{array}$ & 5,348 & $<0.1$ & $<0.1$ & $<0.1$ & $<0.1$ & $\begin{array}{l}< \\
0 \cdot 1\end{array}$ & $\begin{array}{l}(0.0- \\
0.0)\end{array}$ & 31 \\
\hline $\begin{array}{l}\text { Senegal DHS } \\
2016\end{array}$ & 0.9 & $\begin{array}{l}(0 \cdot 7- \\
1 \cdot 2)\end{array}$ & 5,198 & $<0.1$ & $<0.1$ & $<0.1$ & $3 \cdot 2$ & $3 \cdot 2$ & $\begin{array}{l}(1 \cdot 0- \\
9 \cdot 9)\end{array}$ & 46 \\
\hline $\begin{array}{l}\text { Sierra Leone } \\
\text { MIS } 2016\end{array}$ & $54 \cdot 2$ & $\begin{array}{l}(51 \cdot 0- \\
57 \cdot 4)\end{array}$ & 6,440 & $0 \cdot 1$ & $0 \cdot 1$ & $1 \cdot 0$ & $0 \cdot 7$ & $1 \cdot 7$ & $\begin{array}{l}(1 \cdot 3- \\
2 \cdot 3)\end{array}$ & 3491 \\
\hline $\begin{array}{l}\text { Tanzania } \\
\text { AIS/MIS 2011- } \\
12\end{array}$ & $9 \cdot 1$ & $\begin{array}{l}(7 \cdot 8- \\
10 \cdot 6)\end{array}$ & 7,129 & 0.9 & $1 \cdot 2$ & $1 \cdot 7$ & $1 \cdot 6$ & $4 \cdot 5$ & $\begin{array}{l}(3 \cdot 1- \\
6 \cdot 5)\end{array}$ & 649 \\
\hline $\begin{array}{l}\text { Tanzania DHS } \\
\text { 2015-16 }\end{array}$ & $14 \cdot 6$ & $\begin{array}{l}(12 \cdot 6- \\
16 \cdot 8)\end{array}$ & 8,546 & 0.5 & $0 \cdot 7$ & $2 \cdot 1$ & 0.9 & $3 \cdot 4$ & $\begin{array}{l}(2 \cdot 4- \\
5 \cdot 0)\end{array}$ & 1245 \\
\hline $\begin{array}{l}\text { Tanzania MIS } \\
2017\end{array}$ & $7 \cdot 0$ & $\begin{array}{l}(5 \cdot 8- \\
8 \cdot 6)\end{array}$ & 6,433 & $<0.1$ & $<0.1$ & $0 \cdot 2$ & $0 \cdot 7$ & 0.9 & $\begin{array}{l}(0 \cdot 4- \\
2 \cdot 1)\end{array}$ & 453 \\
\hline $\begin{array}{l}\text { Togo DHS } \\
2013-14\end{array}$ & $38 \cdot 5$ & $\begin{array}{l}(35 \cdot 1- \\
42 \cdot 0)\end{array}$ & 2,942 & $1 \cdot 4$ & $0 \cdot 8$ & $3 \cdot 4$ & $0 \cdot 1$ & $4 \cdot 8$ & $\begin{array}{l}(3 \cdot 4- \\
6 \cdot 8)\end{array}$ & 1,132 \\
\hline Togo MIS 2017 & $43 \cdot 9$ & $\begin{array}{l}(39 \cdot 8- \\
48 \cdot 1)\end{array}$ & 2,958 & $0 \cdot 2$ & $0 \cdot 4$ & $0 \cdot 8$ & 0.6 & $1 \cdot 7$ & $\begin{array}{l}(1 \cdot 1- \\
2 \cdot 5)\end{array}$ & 1,299 \\
\hline $\begin{array}{l}\text { Uganda MIS } \\
2014-15\end{array}$ & $32 \cdot 2$ & $\begin{array}{l}(28 \cdot 2- \\
36 \cdot 4)\end{array}$ & 4,347 & $0 \cdot 2$ & 0.5 & $1 \cdot 6$ & $0 \cdot 3$ & $2 \cdot 3$ & $\begin{array}{l}(1 \cdot 4- \\
3 \cdot 6)\end{array}$ & 1,398 \\
\hline $\begin{array}{l}\text { Uganda DHS } \\
2016\end{array}$ & $31 \cdot 1$ & $\begin{array}{l}(28 \cdot 6- \\
33 \cdot 8)\end{array}$ & 4,543 & $0 \cdot 2$ & $3 \cdot 4$ & $5 \cdot 7$ & 0.5 & $6 \cdot 9$ & $\begin{array}{l}(5 \cdot 2- \\
9 \cdot 1)\end{array}$ & 1,414 \\
\hline Total & $26 \cdot 0$ & $\begin{array}{l}(25 \cdot 3- \\
26 \cdot 7)\end{array}$ & 183,265 & 0.5 & $1 \cdot 2$ & $2 \cdot 8$ & 0.9 & $4 \cdot 5$ & $\begin{array}{l}(4 \cdot 1- \\
4 \cdot 8)\end{array}$ & 47,647 \\
\hline
\end{tabular}

\section{Multi-Country Pooled Analysis}

An examination of the distribution of children with at least one severe malaria symptom by background characteristics revealed nearly identical proportions by sex ( $50.3 \%$ of males and $49.7 \%$ of females). The proportion of children with at least one severe malaria symptom decreased with age, ranging from $31.5 \%$ of children age $6-23$ months $(95 \% \mathrm{Cl} 29.0-34.1)$ to $19.9 \%$ of children age $48-59$ months $(95 \% \mathrm{Cl}$ 17.9-22.0). The majority of children symptomatic for severe malaria lived in rural areas (86.8\%,95\% $\mathrm{Cl} 84.1-89.2)$. In addition, a greater proportion of children from households in the lowest wealth quintile $(34.3 \%, 95 \% \mathrm{Cl} 31.3-37.3)$ and in the moderate transmission zone $\mathrm{A}$ 
(37.2\%, 95\% $\mathrm{Cl} 33.4-41.2)$ had at least one symptom of severe malaria compared with those in higher wealth quintiles and other transmission zones. Slightly more than half $(53.9 \%, 95 \% \mathrm{Cl} 49.8-57.9)$ of children with at least one severe malaria symptom were surveyed between 2015 and 2018 (Table 3).

Table 3

Percent distribution of children who had at least one severe malaria symptom among children who were positive for malaria, pooled weighted analysis

\begin{tabular}{|c|c|c|c|}
\hline Background characteristics & Percent Distribution & 95\% Confidence Intervals & Number of children with a malaria infection \\
\hline \multicolumn{4}{|l|}{ Child } \\
\hline \multicolumn{4}{|l|}{ Sex } \\
\hline Male & $50 \cdot 3$ & $(47 \cdot 7-52 \cdot 9)$ & 24,294 \\
\hline Female & $49 \cdot 7$ & $(47 \cdot 1-52 \cdot 3)$ & 23,353 \\
\hline \multicolumn{4}{|l|}{ Age Group (months) } \\
\hline $6-23$ & $31 \cdot 5$ & $(29 \cdot 0-34 \cdot 1)$ & 12,262 \\
\hline $24-35$ & $25 \cdot 6$ & $(23 \cdot 5-27 \cdot 8)$ & 10,669 \\
\hline $36-47$ & $23 \cdot 0$ & $(21 \cdot 1-25 \cdot 1)$ & 12,098 \\
\hline $48-59$ & $19 \cdot 9$ & $(17 \cdot 9-22 \cdot 0)$ & 12,619 \\
\hline \multicolumn{4}{|l|}{ Household } \\
\hline \multicolumn{4}{|l|}{ Place of Residence } \\
\hline Rural & $86 \cdot 8$ & $(84 \cdot 1-89 \cdot 2)$ & 40,818 \\
\hline Urban & $13 \cdot 2$ & $(10 \cdot 8-15 \cdot 9)$ & 6,829 \\
\hline \multicolumn{4}{|l|}{ Wealth Quintile } \\
\hline Lowest & $34 \cdot 3$ & $(31 \cdot 3-37 \cdot 3)$ & 15,302 \\
\hline Second & $28 \cdot 5$ & $(26 \cdot 1-31 \cdot 0)$ & 13,510 \\
\hline Middle & $20 \cdot 3$ & $(18 \cdot 2-22 \cdot 7)$ & 10,254 \\
\hline Fourth & $14 \cdot 3$ & $(12 \cdot 3-16 \cdot 6)$ & 6,518 \\
\hline Highest & $2 \cdot 6$ & $(1 \cdot 8-3 \cdot 6)$ & 2,063 \\
\hline \multicolumn{4}{|l|}{ Malaria Endemicity } \\
\hline Low transmission $<10 \%$ & $14 \cdot 3$ & $(11 \cdot 9-17 \cdot 1)$ & 5,507 \\
\hline Moderate transmission A $11 \%-30 \%$ & $37 \cdot 2$ & $(33 \cdot 4-41 \cdot 2)$ & 18,531 \\
\hline Moderate transmission B 31\%-50\% & $28 \cdot 6$ & $(25 \cdot 0-32 \cdot 4)$ & 17,760 \\
\hline High transmission $>50 \%$ & $19 \cdot 9$ & $(16 \cdot 5-23 \cdot 8)$ & 5,849 \\
\hline \multicolumn{4}{|l|}{ Survey timing } \\
\hline $2011-2014$ & $46 \cdot 1$ & $(42 \cdot 1-50 \cdot 2)$ & 15,977 \\
\hline $2015-2018$ & $53 \cdot 9$ & $(49 \cdot 8-57 \cdot 9)$ & 31,670 \\
\hline Total & $100 \cdot 0$ & & 47,647 \\
\hline
\end{tabular}

\section{Regression Analysis}

The regression analysis shows the odds of reporting at least one symptomatic marker of severe malaria in relation to the different background characteristics of the children. Compared to children age 6-23 months, children age 36-47 months (AOR 0.73, 95\% Cl 0.650.82 ) and $48-59$ months (AOR $0.58,95 \% \mathrm{Cl} 0.51-0.66$ ) were significantly less likely to report at least one symptomatic marker for severe 
malaria. Urban children were significantly more likely to report at least one symptomatic marker for severe malaria as compared to rural children (AOR 1.28, 95\% Cl 1.12-1.48). Socioeconomic status of the household is associated with the likelihood of reporting a severe malaria symptom, with children in the highest wealth quintile having lower odds compared to those from households in the lowest quintile (AOR 0.56, 95\% Cl 0.42-0.76). Malaria endemicity defined by different transmission zones was not associated with the likelihood of reporting a symptom of severe malaria. Finally, the children surveyed between 2015-2018 were significantly less likely to report at least one symptomatic marker for severe malaria compared to children surveyed between 2011-2014 (AOR 0.72, 95\% Cl 0.62-0.83) (Table 4).

Table 4

Multilevel unweighted mixed effects logistic regression model of children who had at least one symptomatic marker for severe malaria among children who were positive for malaria, pooled analysis

\begin{tabular}{|c|c|c|}
\hline Background Characteristic & Adjusted Odd Ratio & $95 \%$ Confidence Intervals \\
\hline \multicolumn{3}{|l|}{ Child } \\
\hline \multicolumn{3}{|l|}{ Sex } \\
\hline Male & \multicolumn{2}{|l|}{1 (Reference) } \\
\hline Female & 0.95 & $0.87-1.04$ \\
\hline \multicolumn{3}{|l|}{ Age Group (months) } \\
\hline $6-23$ & \multicolumn{2}{|l|}{1 (Reference) } \\
\hline $24-35$ & 0.91 & $0 \cdot 81-1 \cdot 02$ \\
\hline $36-47$ & $0 \cdot 73^{\star \star \star}$ & $0.65-0.82$ \\
\hline $48-59$ & $0 \cdot 58 * \star \star$ & $0.51-0.66$ \\
\hline \multicolumn{3}{|l|}{ Household } \\
\hline \multicolumn{3}{|l|}{ Place of Residence } \\
\hline Rural & \multicolumn{2}{|l|}{1 (Reference) } \\
\hline Urban & $1 \cdot 28^{* * *}$ & $1 \cdot 12-1 \cdot 48$ \\
\hline \multicolumn{3}{|l|}{ Wealth Quintile } \\
\hline Lowest & \multicolumn{2}{|l|}{1 (Reference) } \\
\hline Second & $1 \cdot 01$ & $0 \cdot 90-1 \cdot 13$ \\
\hline Middle & 0.91 & $0.80-1 \cdot 03$ \\
\hline Fourth & 0.99 & $0 \cdot 85-1 \cdot 14$ \\
\hline Highest & 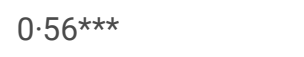 & $0.42-0.76$ \\
\hline \multicolumn{3}{|l|}{ Malaria Endemicity } \\
\hline Low Transmission $<10 \%$ & \multicolumn{2}{|l|}{1 (Reference) } \\
\hline Moderate Transmission A 11\%-30\% & $1 \cdot 01$ & $0 \cdot 86-1 \cdot 19$ \\
\hline Moderate Transmission B 31\%-50\% & 0.99 & $0 \cdot 81-1 \cdot 22$ \\
\hline High Transmission > 50\% & $1 \cdot 06$ & $0 \cdot 83-1 \cdot 34$ \\
\hline \multicolumn{3}{|l|}{ Survey timing } \\
\hline $2011-2014$ & \multicolumn{2}{|l|}{1 (Reference) } \\
\hline $2015-2018$ & $0 \cdot 72^{\star \star \star}$ & $0.62-0.83$ \\
\hline \multicolumn{3}{|c|}{ Level of statistical significance ${ }^{* \star *} p<0.001,{ }^{* *} p<0.01, * p<0.05$} \\
\hline
\end{tabular}

\section{Discussion}


Despite improvements in the diagnosis and documentation of severe malaria cases, there remains uncertainty about the burden of severe malaria cases at the population level. Here we present a comprehensive estimate of severe malaria cases in children from 19 malariaendemic countries in SSA. Our estimates are based on data from population-based household surveys that allow severe malaria cases outside of the formal healthcare system to be directly captured in estimates.

The prevalence estimate of severe malaria in this study ( $4.5 \%$ of malaria-infected children) is consistent with other estimates of severe malaria. This acknowledges that other estimates of severe malaria cases account only for children who access the formal healthcare system [1, 12]. Household surveys test all children age 6-59 months for malaria, and while some of the children who were showing signs of severe illness would have eventually accessed the formal healthcare system, some of these children would have died, recovered at home, or received care outside of the formal healthcare system [7, 9, 12-14]. Estimating severe malaria through household surveys provides countries with a standardized estimate of severe malaria that is comparable across time as well with other countries.

Findings from this study confirm previous observations that severe malaria is dependent on age and transmission intensity [5-8]. Younger children were significantly more likely to have severe malaria, and although not significant, the risk of severe malaria was greater in high malaria transmission zones. However, unlike previous research, we did not find an interaction between age and the intensity of malaria transmission in relation to having at least one symptom of severe malaria (data not shown) [5]. One explanation is the limited age range of children (6-59 months) in this analysis. Past studies that have examined the association of variations in age and endemicity on the clinical manifestation of severe malaria included children up to age $10[5,6,8]$.

Children surveyed between 2015-2018 were significantly less likely to have severe malaria symptoms as compared to children surveyed between 2011-2015. This is controlling for malaria transmission level and is irrespective of variations in malaria prevalence since all children included in the analysis were positive for malaria, according to RDT. This finding aligns with the 2019 World Malaria Report, which reported a decrease in malaria deaths since 2010 [1]. While the role of malaria control interventions in this difference cannot be assumed, since 2015, there has been an increase in the number of malaria interventions in SSA, including the implementation of seasonal malaria chemoprevention and universal coverage of insecticide-treated nets [1]. The impact of these interventions on severe malaria cases needs further exploration.

This analysis also indirectly highlights potential variation in care-seeking patterns for severe malaria cases across SSA. Urban children were significantly more likely to have at least one severe malaria symptom as compared to rural children despite a higher prevalence of severe malaria among the rural population. In addition, the country was highly significant in the model even when controlling for malaria endemicity. By examining severe malaria cases at the household level, this analysis is more likely to include children whose caregivers have taken them to a healthcare facility but whose illness did not resolve or have not sought care for the child's illness. While the decision to seek care is ultimately decided by the caregiver, it is highly influenced by factors such as the availability of government-based facilities, country wealth, cost of care, and education[14, 20-23]. Further exploration is needed into country and urban-rural variations in care-seeking and its association with severe malaria burden estimates.

This study has a several limitations. Children with severe malaria frequently develop one or more complications, including severe anemia, respiratory distress, or cerebral malaria. This analysis examined children who had at least one symptomatic marker for severe malaria. We did not disaggregate this analysis by proxies for respiratory distress or cerebral malaria. Examining severe anemia is possible because this is a discrete diagnosis based on hemoglobin levels. However, to fully disaggregate by respiratory distress or cerebral malaria would require additional questions about symptoms such as prostration and the number and severity of convulsions [2].

The use of household-level data is a noteworthy advantage of this study, but it also introduces a principal limitation. There is a risk of including uncomplicated malaria cases or non-malaria cases in our proxy definition. Malaria positivity is based on RDT-detectable antigens that continue to circulate in the blood after the infection has cleared, and severe malaria symptoms are non-specific [24]. Our definition of severe malaria relies on caregiver self-report rather than a diagnosis by a clinician at a health facility. Although the interviewer for the biomarker questionnaire is a trained biomarker health technician (usually a nurse), which may improve the questionnaire responses, the non-specific nature of severe malaria remains an issue. We have addressed this limitation by narrowing the proxy definition of severe malaria to only examine loss of consciousness, rapid breathing, seizures, or severe anemia (hemoglobin $<5 \mathrm{~g} / \mathrm{dL}$ adjusted for altitude). These symptoms are more distinct than some other symptoms (extreme weakness and heart problems) caregivers are asked and most closely align with the WHO clinical manifestations of severe malaria. We assumed these symptoms would not be confused by caregivers, even those with a limited education. However, we were unable to examine the reliability of reported signs and symptoms because caregivers were not asked questions on severe malaria symptoms for malaria negative children. In addition, by limiting our definition of severe malaria symptoms, there is the possibility that we may be missing cases. 
This analysis only includes children with malaria according to RDT, which further minimizes the possibility that the child is sick with an illness other than severe malaria. However, as noted above, there is still a risk of including non-malaria cases in our proxy definition because malaria positivity is based on RDT-detectable antigen that circulates in the blood after the infection has cleared. More sensitive measures of malaria diagnosis than standard HRP-II RDTs should be explored, such as microscopy, highly sensitive RDTs, or polymerase chain reaction (PCR).

\title{
Conclusions
}

This analysis investigated severe malaria symptoms in children age 6-59 months who are positive for malaria as identified in populationbased surveys. To date, there has been a gap in knowledge about the burden of severe malaria at the population level since previous estimates have relied exclusively on data from formal healthcare services. This analysis presents the most comprehensive estimate of the prevalence of severe malaria in children age 6-59 months from 19 countries across multiple malaria endemicity zones. The data in this analysis were initially collected for severe malaria case referral purposes, but also provide invaluable insights into severe malaria cases at the population level.

\author{
Abbreviations \\ DHS Demographic and Health Survey \\ $\mathrm{g} / \mathrm{dL}$ grams per deciliter \\ HRP-II histidine-rich protein II \\ MIS Malaria Indicator Survey \\ PCR polymerase chain reaction \\ P. falciparum Plasmodium falciparum \\ RDT rapid diagnostic test \\ SSA sub-Saharan Africa \\ WHO World Health Organization
}

\section{Declarations}

\section{Ethics approval and consent to participate}

Not applicable

\section{Consent for publication}

Not applicable

\section{Availability of data and material}

The datasets analyzed during the current study are available from The DHS Program web site, www.dhsprogram.com.

\section{Competing interests}

Not applicable

\section{Funding-}


Cameron Taylor, Sorrel Namaste, Joanna Lowell and Johanna Useem were supported by the United States Agency for International Development (USAID) through The DHS Program (\#720-OAA-18C-00083). Yazoume Ye was supported through the USAID and PMI under the terms of the PMI Measure Malaria Associate Award No. 7200AA19LA00001. PMI Measure Malaria is implemented by the University of North Carolina at Chapel Hill, in partnership with ICF Macro, Inc.; Tulane University; John Snow, Inc.; and Palladium International, LLC.

\section{Author's Contributions-}

$\mathrm{CT}, \mathrm{SN}$, and JL conceived of and designed the study. CT and JU assisted in the collection of study variables. CT performed all data analysis with $\mathrm{SN}$ and $\mathrm{YY}$ contributing to the interpretation. CT wrote the manuscript with inputs from SN, JL, JU, and YY. All authors read and approved the final manuscript.

\section{Acknowledgments-}

The authors would like to thank Tom Fish, The DHS Program geospatial analyst, for help in preparing the $\operatorname{PfPR}_{2-10}$ data for merging as well as the creation of the analysis map. The authors would also like to thank Diane Stoy for editorial review and Annette McFarland for assisting in the creation of graphics. Additional thanks go to Jordan Burns, Misun Choi, Lia Florey, Kevin Griffith, Kathryn Hogan, and Meera Venkatesan of PMI at USAID for their invaluable feedback on this analysis. The authors would like to acknowledge Bernard Barrère (1953-2018), who diligently served The DHS Program for 29 years in various capacities. Bernard was the impetus for the inclusion of severe malaria questions in 2011 as part of the severe malaria referral process. We hope that this analysis extends the impact that Bernard had on the world through his life of service to global health and data.

\section{References}

1. World Health Organization. World Malaria Report. vol. licen. Geneva: World Health Organization; 2019.

2. World Health Organization. Management of severe malaria - A practical handbook. Third edition. 2013.

3. Malaria. Fact Sheet https://www.who.int/news-room/fact-sheets/detail/malaria.

4. Bejon P, Berkley JA, Mwangi T, Ogada E, Mwangi I, Maitland K, Williams T, Scott JAG, English M, Lowe BS, et al. Defining Childhood Severe Falciparum Malaria for Intervention Studies. PLOS Medicine. 2007;4:e251.

5. Okiro EA, Al-Taiar A, Reyburn H, Idro R, Berkley JA, Snow RW. Age patterns of severe paediatric malaria and their relationship to Plasmodium falciparum transmission intensity. Malaria Journal. 2009;8:4.

6. Carneiro I, Roca-Feltrer A, Griffin JT, Smith L, Tanner M, Schellenberg JA, Greenwood B, Schellenberg D. Age-Patterns of Malaria Vary with Severity, Transmission Intensity and Seasonality in Sub-Saharan Africa: A Systematic Review and Pooled Analysis. PLOS ONE. 2010;5:e8988.

7. Severe malaria. Trop Med Int Health 2014, 19 Suppl 1:7-131.

8. Roca-Feltrer A, Carneiro I, Smith L, Schellenberg JRMA, Greenwood B, Schellenberg D. The age patterns of severe malaria syndromes in sub-Saharan Africa across a range of transmission intensities and seasonality settings. Malaria Journal. 2010;9:282.

9. Camponovo F, Bever CA, Galactionova K, Smith T, Penny MA. Incidence and admission rates for severe malaria and their impact on mortality in Africa. Malar J. 2017;16:1-1.

10. Ye Y, Kyobutungi C, Ogutu B, Villegas L, Diallo D, Tinto H, Oduro A, Sankoh O. Malaria mortality estimates: need for agreeable approach. Tropical Med Int Health. 2013;18:219-21.

11. Mathers CD, Fat DM, Inoue M, Rao C, Lopez AD. Counting the dead and what they died from: an assessment of the global status of cause of death data. Bull World Health Organ. 2005;83:171-7.

12. Roca-Feltrer A, Carneiro I, Armstrong Schellenberg JR. Estimates of the burden of malaria morbidity in Africa in children under the age of 5 years. Trop Med Int Health. 2008;13:771-83.

13. Reyburn H, Mbatia R, Drakeley C, Bruce J, Carneiro I, Olomi R, Cox J, Nkya WM, Lemnge M, Greenwood BM, Riley EM. Association of transmission intensity and age with clinical manifestations and case fatality of severe Plasmodium falciparum malaria. Jama. 2005;293:1461-70.

14. Alegana VA, Maina J, Ouma PO, Macharia PM, Wright J, Atkinson PM, Okiro EA, Snow RW, Tatem AJ. National and sub-national variation in patterns of febrile case management in sub-Saharan Africa. Nat Commun. 2018;9:4994.

15. Survey Types [https://dhsprogram.com/What-We-Do/Survey-Types/index.cfm].

Page $12 / 13$ 
16. Malaria Indicator Survey Toolkit [https:// ].

17. Croft TN, Marshall AMJ. CK A: Guide to DHS Statistics. Rockville: ICF; 2018.

18. Rutstein SO. K. J: The DHS Wealth Index. Calverton. Maryland: ORC Macro; 2004.

19. Pfeffer DA, Lucas TCD, May D, Harris J, Rozier J, Twohig KA, Dalrymple U, Guerra CA, Moyes CL, Thorn M, et al. malariaAtlas: an R interface to global malariometric data hosted by the Malaria Atlas Project. Malaria Journal. 2018;17:352.

20. Battle KE, Bisanzio D, Gibson HS, Bhatt S, Cameron E, Weiss DJ, Mappin B, Dalrymple U, Howes RE, Hay SI, Gething PW. Treatmentseeking rates in malaria endemic countries. Malar J. 2016;15:20-0.

21. El-Houderi A, Constantin J, Castelnuovo E, Sauboin C. Economic and Resource Use Associated With Management of Malaria in Children Aged < 5 Years in Sub-Saharan Africa: A Systematic Literature Review. MDM Policy Practice. 2019;4:2381468319893986.

22. Issifou S, Kendjo E, Missinou M, Matsiegui P, Dzeing-Ella A, Dissanami F, Kombila M, Krishna S, Kremsner P. Differences in Presentation of Severe Malaria in Urban and Rural Gabon. Am J Trop Med Hyg. 2007;77:1015-9.

23. Sumbele IUN, Kimbi HK, Ndamukong-Nyanga JL, Nweboh M, Anchang-Kimbi JK, Lum E, Nana Y, Ndamukong KKJ, Lehman LG. Malarial Anaemia and Anaemia Severity in Apparently Healthy Primary School Children in Urban and Rural Settings in the Mount Cameroon Area: Cross Sectional Survey. PLOS ONE. 2015;10:e0123549.

24. Dalrymple U, Arambepola R, Gething PW, Cameron E. How long do rapid diagnostic tests remain positive after anti-malarial treatment? Malaria Journal. 2018;17:228.

\section{Figures}

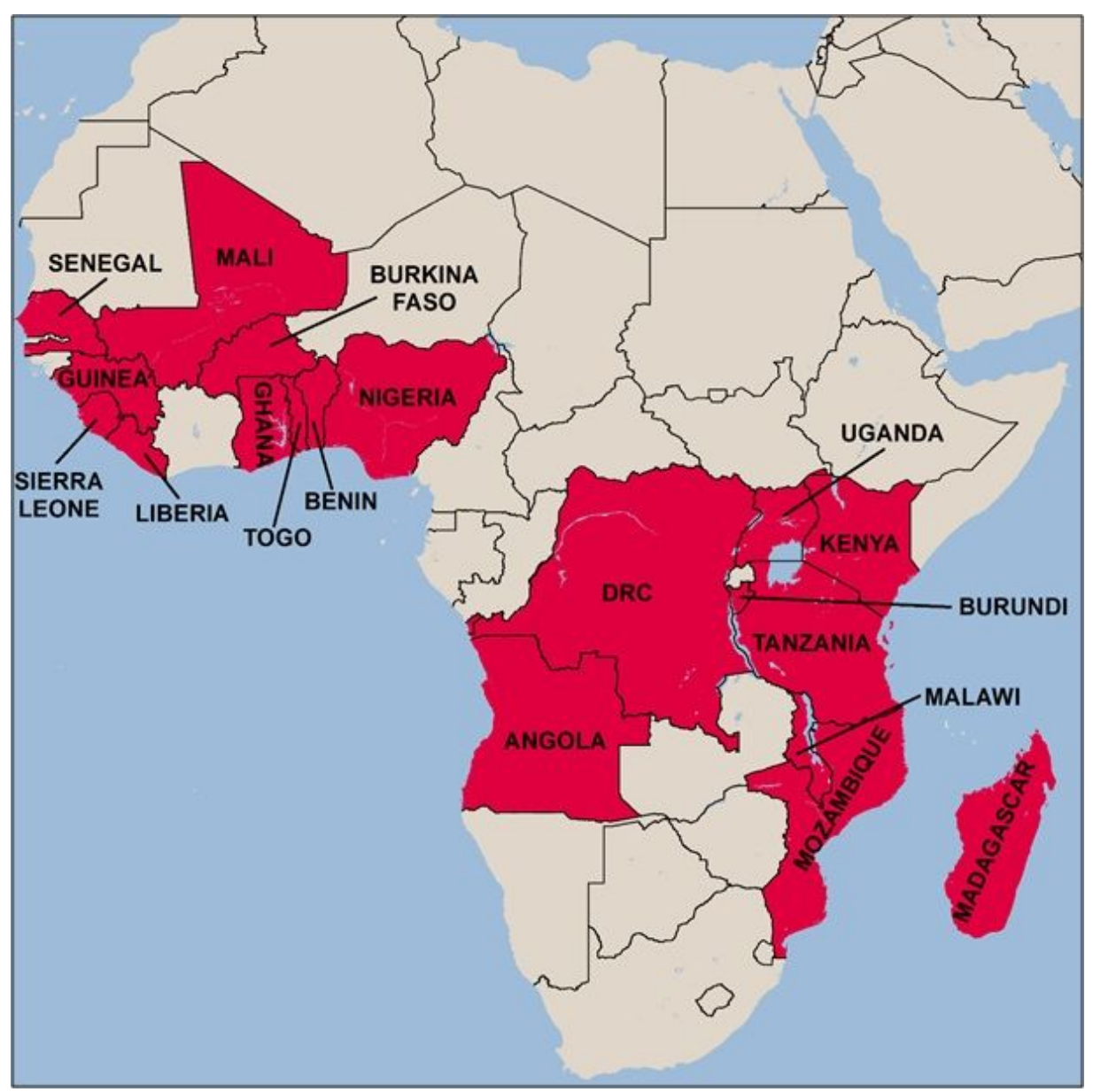

\section{Figure 1}

Map of countries included in the study 